\title{
Regularities and Their Relations to Error Bounds*
}

\author{
Kung Fu Ng ${ }^{\dagger}$ and Wei Hong Yang ${ }^{\ddagger}$
}

\begin{abstract}
In this paper, we mainly study various notions of regularity for a finite collection $\left\{C_{1}, \cdots, C_{m}\right\}$ of closed convex subsets of a Banach space $X$ and their relations with other fundamental concepts. We show that a proper lower semicontinuous function $f$ on $X$ has a Lipschitz error bound (resp., $\Upsilon$-error bound) if and only if the pair $\{\operatorname{epi}(f), X \times\{0\}\}$ of sets in the product space $X \times \mathbb{R}$ is linearly regular (resp., regular). Similar results for multifunctions are also established. Next, we prove that $\left\{C_{1}, \cdots, C_{m}\right\}$ is linearly regular if and only if it has the strong CHIP and the collection $\left\{N_{C_{1}}(z), \cdots, N_{C_{m}}(z)\right\}$ of normal cones at $z$ has property $(\mathrm{G})$ for each $z \in C:=\cap_{i=1}^{m} C_{i}$. Provided that $C_{1}$ is a closed convex cone and that $C_{2}=Y$ is a closed vector subspace of $X$, we show that $\left\{C_{1}, Y\right\}$ is linearly regular if and only if there exists $\alpha>0$ such that each positive (relative to the order induced by $C_{1}$ ) linear functional on $Y$ of norm one can be extended to a positive linear functional on $X$ with norm bounded by $\alpha$. Similar characterization is given in terms of normal cones.
\end{abstract}

Key words. epigraph, error bound, graph, linearly regular, multifunction, normal cone, property $(\mathrm{G})$, regular, strong CHIP, tangent cone, positive linear functional

AMS Subject Classifications. 90C25,90C31,49J52,46A40

\section{Introduction}

The main objective of this paper is to study various notions of regularity (for definitions, see Section 2) for a finite collection $\left\{C_{1}, \cdots, C_{m}\right\}$ of closed convex subsets of a Banach space $X$ and their relations with other fundamental concepts such as the strong conical hull intersection property (the strong CHIP for short), Jameson's (G)-property and error bounds in mathematical programming. The concept of regularity and its quantitative versions were introduced in $[3,4,6]$ by Bauschke and Borwein, and were utilized to establish norm or linear convergence results. The concepts of the strong CHIP was introduced by Deutsch, Li and Ward in [8], and was utilized in [9] to reformulate certain optimization problems with constraints. All the works cited above were in the Hilbert space or Euclidean space setting. The concept of property $(G)$ was introduced by Jameson in [13] for the case when each $C_{i}$ is a cone, and was utilized to give a duality charaterization of the linear regularity. His result is extended here to the general case (i.e. without the additional assumption that each $C_{i}$ is a cone). More direct stimulus to our work is the

\footnotetext{
*This research was supported by an Earmarked grant from the Research Grant Council of Hong Kong

${ }^{\dagger}$ Department of Mathematics, The Chinese University of Hong Kong, Shatin, New Territory, Hong Kong (kfng@math.cuhk.edu.hk)

${ }^{\ddagger}$ Department of Mathematics, The Chinese University of Hong Kong, Shatin, New Territory, Hong Kong (whyang@math.cuhk.edu.hk)
} 
paper [6] by Bauschke, Borwein and Li, where all these notions were studied with many interesting results on the relationships among them. Assuming $C:=\cap_{i=1}^{m} C_{i} \neq \emptyset$, we show in Theorem 4.2 that $\left\{C_{1}, \cdots, C_{m}\right\}$ is linearly regular if and only if it has the strong CHIP and the collection $\left\{N_{C_{1}}(z), \cdots, N_{C_{m}}(z)\right\}$ of normal cones at $z$ has property $(\mathrm{G})$ for each $z \in C$. For the special case when $X$ is an Euclidean space, some partial results are known before: the sufficient part was proved in [6, Corollary 4] while the assertion that the strong CHIP follows from the linear regularity was observed by Pang in [21, Proposition 6] (see also [14, Proposition 2] and [6, Theorem 3]). Provided that $C_{1}$ is a closed convex cone and that $C_{2}=Y$ is a closed vector subspace of $X$, we show in Theorem 4.3 that $\left\{C_{1}, Y\right\}$ is linearly regular if and only if there exists $\alpha>0$ such that each positive (relative to the order induced by $C_{1}$ ) linear functional on $Y$ of norm one can be extended to a positive linear functional on $X$ with norm bounded by $\alpha$. Similar characterization is given in terms of normal cones.

The definition of regularities can obviously be extended to the case where each $C_{i}$ is not necessarily convex. Nevertheless, this naive extension of the definition enables us to establish another interesting link of the concept of regularity to the study of error bounds for functions which are not necessarily convex. We show in Section 3 that a proper lower semicontinuous function $f$ on $X$ has a Lipschitz error bound (resp., $\Upsilon$-error bound) if and only if the pair $\{\operatorname{epi}(f), X \times\{0\}\}$ of sets in the product space $X \times \mathbb{R}$ is linearly regular (resp., regular). Similar results for multifunctions are also established. In an accompanying paper [20] further applications will be made in this direction; in particular we give a complete answer to the error bound problem for the inequality system $A x+b \in S_{\text {ice }}$, where $A: X \rightarrow \mathbb{R}^{n}$ is a continuous linear operator, $b \in \mathbb{R}^{n}$ and $S_{\text {ice }}$ denotes the "ice-cream cone" consisting of all $\left(x_{1}, \cdots, x_{n}\right) \in \mathbb{R}^{n}$ satisfying $\sqrt{\sum_{i=1}^{n-1} x_{i}^{2}} \leq x_{n}$.

The authors would like to thank Professor Jong-Shi Pang for valuable suggestions who first suggested us to study the error bound problem involving the ice-cream cone; our study of this problem and his paper [21] soon led us to address issues relating to regularity and error bounds. We also thank the two referees and the associate editor: their comments have helped to improve our presentation, and their criticisms have prompted us to try to place our results in a more motivated perspective relating to previous published works. In particular the whole Section 4 is added to in this spirit in the revision.

\section{Definitions and Preliminary Results}

Let $X$ be a Banach space (unless specified otherwise). Let $X^{*}$ denote the (topological) dual of $X$ with the norm \|\|$_{*}$ defined by

$$
\left\|x^{*}\right\|_{*}=\sup \left\{\left|x^{*}(x)\right|: x \in X,\|x\| \leq 1\right\}, \quad x^{*} \in X^{*} .
$$

Following the usual practice, we often write \|\| for \|\|$_{*}$. For a set $S$ in $X$, the closure (resp. boundary) of $S$ is denoted by $\bar{S}$ (resp. bd $S$ ). Moreover, $\iota_{S}$ denotes the indicator function of $S: \iota_{S}(x)=0$ if $x \in S$ and $\iota_{S}(x)=+\infty$ if $x \notin S$. The polar of a set $S$ is

$$
S^{\circ}=\left\{x^{*} \in X^{*}: x^{*}(x) \leq 1, \forall x \in S\right\}
$$


and the bipolar (cf. [12, p.67]) of $S$ is

$$
{ }^{\circ}\left(S^{\circ}\right):=\left\{x \in X: x^{*}(x) \leq 1, \forall x^{*} \in S^{\circ}\right\} .
$$

Note that if $S$ is a cone then $S^{\circ}=\left\{x^{*} \in X^{*}: x^{*}(x) \leq 0, \forall x \in S\right\}$ and ${ }^{\circ}\left(S^{\circ}\right)=\{x \in X$ : $\left.x^{*}(x) \leq 0, \forall x^{*} \in S^{\circ}\right\}$. For a convex set $S$ and $x \in S, T_{S}(x)$ denotes the tangent cone of $S$ at $x$ and is defined by (cf. [2, p.166])

$$
T_{S}(x):=\overline{\left(\cup_{t>0} \frac{1}{t}(S-x)\right)} .
$$

It is easy to see that $(2.2)$ can be refomulated as the following

$$
T_{S}(x)=\{h \in X: \operatorname{dist}(x+t h, S)=o(t) \text { for } t \geq 0\} .
$$

Here and throughout, $\operatorname{dist}(x, S)$ denotes the distance of $x$ to the set $S$. The normal cone of $S$ at $x$ is denoted by $N_{S}(x)$ and is defined to be the polar of $T_{S}(x)$ :

$$
N_{S}(x)=\left\{x^{*} \in X^{*}: x^{*}(h) \leq 0, \forall h \in T_{S}(x)\right\} .
$$

By the Bipolar Theorem (cf. [12, p.68]), $T_{S}(x)$ is the bipolar of itself.

Following [8, 9], a colletion $\left\{C_{i}\right\}_{i=1}^{m}$ of closed subsets of $X$ is said to have strong CHIP at $x \in \cap_{i=1}^{m} C_{i}$ if $N_{C}(x)=\sum_{i=1}^{m} N_{C_{i}}(x)$.

Lemma 2.1. Let $X$ be a normed vector space, $K \subset X$ a nonempty closed convex set, $y \in K$ and $h \in X \backslash T_{K}(y)$. Let $x(t)=y+$ th for $t>0$. Then

$$
\lim _{t \rightarrow 0} \frac{\operatorname{dist}\left(x(t), y+T_{K}(y)\right)}{\operatorname{dist}(x(t), K)}=1 .
$$

Proof. Since $K \subseteq y+T_{K}(y)$, we have $0<\operatorname{dist}\left(x(t), y+T_{K}(y)\right) \leq \operatorname{dist}(x(t), K)$. Thus we only need to prove $\liminf _{t \rightarrow 0} \frac{\operatorname{dist}\left(x(t), y+T_{K}(y)\right)}{\operatorname{dist}(x(t), K)} \geq 1$, namely

$$
\liminf _{t \rightarrow 0} \frac{t \operatorname{dist}\left(h, T_{K}(y)\right)}{\operatorname{dist}(x(t), K)} \geq 1 \text {. }
$$

Let $\epsilon>0$. Take $\bar{h} \in T_{K}(y)$ such that

$$
\|h-\bar{h}\| \leq \operatorname{dist}\left(h, T_{K}(y)\right)+\epsilon .
$$

For $t>0$, let $z(t)=y+t \bar{h}$. Since $\bar{h} \in T_{K}(y)$, we have $\operatorname{dist}(z(t), K)=o(t)$. Thus

$$
\operatorname{dist}(x(t), K) \leq\|x(t)-z(t)\|+o(t)=t\|h-\bar{h}\|+o(t) .
$$

It follows that

$$
\frac{\operatorname{tdist}\left(h, T_{K}(y)\right)}{\operatorname{dist}(x(t), K)} \geq \frac{\operatorname{tdist}\left(h, T_{K}(y)\right)}{t\left[\operatorname{dist}\left(h, T_{K}(y)\right)+\epsilon\right]+o(t)} .
$$

Hence (2.6) is seen to hold as $\epsilon>0$ is arbitrary. 
Lemma 2.2. Let $X$ be a Banach space, $K \subset X$ a nonempty closed convex set. Let $0<\gamma<1$. Then for each $x \in X$ there exists $z \in K$ such that

$$
\operatorname{dist}\left(x, z+T_{K}(z)\right) \geq \gamma \operatorname{dist}(x, K) .
$$

Proof. Let $x \notin K$. By [19, Proposition 1.3], there exist $z \in K$ and $z^{*} \in X^{*}$ with norm 1 supporting $K$ at $z$ such that

$$
z^{*}(x-z) \geq \gamma\|x-z\|
$$

Note in particular that $z^{*}(y) \leq 0$ for each $y \in T_{K}(z)$. Denoting $\left\{x \in X: z^{*}(x)=0\right\}$ by $\operatorname{Ker}\left(z^{*}\right)$, it follows that

$$
\operatorname{dist}\left(x, z+T_{K}(z)\right) \geq \operatorname{dist}\left(x-z, \operatorname{Ker}\left(z^{*}\right)\right)=z^{*}(x-z) \geq \gamma\|x-z\| \geq \gamma \operatorname{dist}(x, K) .
$$

For a real number $\alpha$, we write $\alpha_{+}$for $\max \{\alpha, 0\}$. Given lower semicontinuous convex functions $f$ and $g$ on $X$, the conjugate function of $f$ is denoted by $f^{*}$, and the infimal convolution of $f, g$ is denoted by $f \square g$ :

$$
(f \square g)(x)=\inf _{u+v=x}\{f(u)+g(v)\}, \quad \forall x \in X .
$$

If the infimum is attained, the convolution is said to be exact. Denote $\left\{x^{*} \in X^{*}\right.$ : $\left.f^{*}\left(x^{*}\right)<+\infty\right\}$ by $\operatorname{dom}\left(f^{*}\right)$. We shall need the following results from convex analysis.

Lemma 2.3. Let $f$ and $g$ be proper lower semicontinuous convex functions on $X$.

(i) $(f \square g)^{*}=f^{*}+g^{*}$.

(ii) $f \leq g \Leftrightarrow g^{*} \leq f^{*}$.

(iii) If $p, q>1$ and $\frac{1}{p}+\frac{1}{q}=1$, then $\left(\frac{1}{p}\|\cdot\|^{p}\right)^{*}=\frac{1}{q}\|\cdot\|_{*}^{q}$.

(iv) If $C$ is a closed convex cone in $X$, then $\left(\iota_{C}\right)^{*}=\iota_{C}$.

(v) For any $\alpha>0$ and any $x^{*} \in X^{*},(\alpha f)^{*}\left(x^{*}\right)=\alpha f^{*}\left(\frac{x^{*}}{\alpha}\right)$.

(vi) If at least one of $f, g$ is continuous, then $(f+g)^{*}=f^{*} \square g^{*}$ and

$$
\left(f^{*} \square g^{*}\right)\left(x^{*}\right)=\min _{u^{*}+v^{*}=x^{*}}\left\{f\left(u^{*}\right)+g\left(v^{*}\right)\right\}, \quad \forall x^{*} \in \operatorname{dom}\left(f^{*}\right)+\operatorname{dom}\left(g^{*}\right) .
$$

Proof. For (vi), see [1, p.127] and [2, Chap. 4, Sec. 4, Corollary 12]. For other parts, see [2, Chap. 4] and [22, Chap. 16].

Remark 2.1. By Lemma 2.3 (vi), if $f_{i}$ is continuous on $X$ for each $1 \leq i \leq m$, then

$$
\left(f_{1}+\cdots+f_{m}\right)^{*}\left(x^{*}\right)=\left(f_{1}^{*} \square \cdots \square f_{m}^{*}\right)\left(x^{*}\right)=\min _{\sum_{i=1}^{m} x_{i}^{*}=x^{*}}\left\{\sum_{i=1}^{m} f_{i}^{*}\left(x_{i}^{*}\right)\right\}, \quad \forall x^{*} \in \sum_{i=1}^{m} \operatorname{dom}\left(f_{i}^{*}\right) .
$$


In the proof of our Theorem 4.1, we need the following elementary lemma. For $x \in \mathbb{R}^{m}$, let $x_{i}$ denote the i-th coordinate of $x$ for $1 \leq i \leq m$. For $1 \leq p \leq \infty$, The p-norm of $x$, denoted by $\|x\|_{p}$, is defined by $\|x\|_{p}=\left[\sum_{i=1}^{m}\left|x_{i}\right|^{p}\right]^{\frac{1}{p}}$, where we adopt the convention that, for $p=\infty$,

$$
\max \left\{\left|x_{1}\right|, \cdots,\left|x_{m}\right|\right\}:=\left[\sum_{i=1}^{m}\left|x_{i}\right|^{p}\right]^{\frac{1}{p}}
$$

Lemma 2.4. $m^{-\frac{1}{p}}\|\cdot\|_{p} \leq\|\cdot\|_{\infty} \leq\|\cdot\|_{p} \leq\|\cdot\|_{1} \leq m^{1-\frac{1}{p}}\|\cdot\|_{p}$ for $p \geq 1$. Moreover, for any $x \in \mathbb{R}^{m},\left\{\|x\|_{p}\right\}$ is converges monotonically to $\|x\|_{\infty}$.

Proof. For a proof, see [10, p.145].

\section{Regularities of Sets in Metric or Normed Spaces}

The results in this section do not require the completeness assumption: For subsection $3.1, X$ is assumed to be a metric space while for subsection $3.2, X$ is assumed to be a normed vector space.

\subsection{Metric Space Case}

Throughout this subsection, let $X$ and $Y$ denote metric spaces. For simplicity of notations, we use the same $d$ to denote the given metrics for $X$ and $Y$, as well as the metric for the product $X \times Y$ defined by

$$
d\left((x, y),\left(x^{\prime}, y^{\prime}\right)\right)=d\left(x, x^{\prime}\right)+d\left(y, y^{\prime}\right) .
$$

The following definitions are taken from $[3,4]$ though, at that time, $X$ was assumed to be a Hilbert space and each $C_{i}$ was assumed to be convex.

Definition 3.1. Let $\left\{C_{i}\right\}_{i=1}^{m}$ be a collection of closed sets in $X$ such that $C:=\cap_{i=1}^{m} C_{i} \neq \emptyset$. We say that $\left\{C_{i}\right\}_{i=1}^{m}$ is

(a) regular if for any sequence $\left\{x_{k}\right\}$ in $X$ it holds that

$$
\max _{1 \leq i \leq m}\left\{\operatorname{dist}\left(x_{k}, C_{i}\right)\right\} \rightarrow 0 \Rightarrow \operatorname{dist}\left(x_{k}, C\right) \rightarrow 0
$$

(b) linearly regular if there exists $\tau>0$ such that

$$
\operatorname{dist}(x, C) \leq \tau \max _{1 \leq i \leq m}\left\{\operatorname{dist}\left(x, C_{i}\right)\right\}, \quad \forall x \in X .
$$

(in this case, we also say that $\left\{C_{i}\right\}$ is linearly regular with modulus $\tau$ ). 
Note that $\tau$ must not be less than 1 because $C \subseteq C_{i}$ for each $i$. Note also that (b) holds if and only if there exist $\kappa>0$ and $p \in[1, \infty]$ such that

$$
\operatorname{dist}(x, C) \leq \kappa\left[\sum_{i=1}^{m} \operatorname{dist}^{p}\left(x, C_{i}\right)\right]^{\frac{1}{p}}, \quad \forall x \in X .
$$

Let $f: X \rightarrow(-\infty,+\infty]$ be a function such that $S_{f}:=\{x \in X: f(x) \leq 0\} \neq \emptyset$. We say that $f$ has an error bound if there exists $\tau>0$ such that $\operatorname{dist}\left(x, S_{f}\right) \leq \tau[f(x)]_{+}$for each $x \in X$ (in this case we also say that $\tau$ is an error bound of $f$ ). In the situation of Definition 3.1, if we define $f$ by

$$
f(x)=\max _{1 \leq i \leq m}\left\{\operatorname{dist}\left(x, C_{i}\right)\right\}, \quad x \in X,
$$

then $C=S_{f}$, and (3.2) simply means that $\tau$ is an error bound of $f$. That is, $\left\{C_{i}\right\}_{i=1}^{m}$ is linearly regular if and only if $f$ defined by (3.4) has an error bound.

For the rest of this section, we shall focus on the special case when $m=2$. Though the order is unimportant, $\left\{C_{1}, C_{2}\right\}$ is referred to as a pair to emphasize that the collection consists of two sets. The following theorem will be useful to us; part (b) reduces the linear regularity of $\left\{C_{1}, C_{2}\right\}$ to a simpler one: the distance from the points of one of the sets (say $C_{1}$ ) to the intersection $C$ are bounded by a constant multiple of their distances to the other set $C_{2}$. Part (a) is of similar spirit as (b).

Theorem 3.1. Let $X$ be a metric space, and let $C_{1}, C_{2}$ be closed sets in $X$ such that $C:=C_{1} \cap C_{2} \neq \emptyset$. Then the following assertions hold.

(a) The pair $\left\{C_{1}, C_{2}\right\}$ is regular if and only if

$$
\lim _{k \rightarrow+\infty} \operatorname{dist}\left(x_{k}, C\right)=0 \text { whenever }\left\{x_{k}\right\} \subseteq C_{1} \text { and } \lim _{k \rightarrow+\infty} \operatorname{dist}\left(x_{k}, C_{2}\right)=0 .
$$

(b) The pair $\left\{C_{1}, C_{2}\right\}$ is linearly regular if and only if there exists $\tau>0$ such that

$$
\operatorname{dist}(x, C) \leq \tau \operatorname{dist}\left(x, C_{2}\right), \quad \forall x \in C_{1} .
$$

In fact if (3.6) holds then

$$
\operatorname{dist}(x, C) \leq(2 \tau+1) \max \left\{\operatorname{dist}\left(x, C_{1}\right), \operatorname{dist}\left(x, C_{2}\right)\right\} \quad \forall x \in X .
$$

Proof. The necessity parts of (a) and (b) are trivial. Conversely suppose (3.5) holds but that $\left\{C_{1}, C_{2}\right\}$ is not regular: there exist $\epsilon>0$ and a sequence $\left\{y_{k}\right\}$ in $X$ such that

$$
\lim _{k \rightarrow+\infty}\left[\max \left\{\operatorname{dist}\left(y_{k}, C_{1}\right), \operatorname{dist}\left(y_{k}, C_{2}\right)\right\}\right]=0
$$

but

$$
\inf _{k} \operatorname{dist}\left(y_{k}, C\right)>\epsilon .
$$

By (3.8), there exists $\left\{z_{k}\right\} \subset C_{1}$ such that $\lim _{k \rightarrow+\infty} d\left(y_{k}, z_{k}\right)=0$ and hence that $\lim _{k \rightarrow+\infty} \operatorname{dist}\left(z_{k}, C_{2}\right)=$ 0 . From (3.9), it follows also that $\operatorname{dist}\left(z_{k}, C\right)>\frac{\epsilon}{2}$ for all large enough $k$. This contradicts $(3.5)$. 
To complete the proof of Theorem 3.1, it remains to show that (3.6) implies (3.7). Suppose (3.7) is false: there exists $\bar{x} \in X$ such that

$$
\operatorname{dist}(\bar{x}, C)>(2 \tau+1) \max \left\{\operatorname{dist}\left(\bar{x}, C_{1}\right), \operatorname{dist}\left(\bar{x}, C_{2}\right)\right\}
$$

Let $\epsilon>0$; take $a_{1} \in C_{1}$ and $a_{2} \in C_{2}$ such that

$$
d\left(\bar{x}, a_{i}\right) \leq \operatorname{dist}\left(\bar{x}, C_{i}\right)+\epsilon, \quad i=1,2 .
$$

Then, it follows from (3.6) that

$$
\begin{aligned}
& \max \left\{\operatorname{dist}\left(\bar{x}, C_{1}\right), \operatorname{dist}\left(\bar{x}, C_{2}\right)\right\}+\epsilon \\
\geq & \max \left\{d\left(\bar{x}, a_{1}\right), d\left(\bar{x}, a_{2}\right)\right\} \\
\geq & \frac{1}{2}\left[d\left(\bar{x}, a_{1}\right)+d\left(\bar{x}, a_{2}\right)\right] \\
\geq & \frac{1}{2} d\left(a_{1}, a_{2}\right) \geq \frac{1}{2} \operatorname{dist}\left(a_{1}, C_{2}\right) \geq \frac{1}{2 \tau} \operatorname{dist}\left(a_{1}, C\right) \\
\geq & \frac{1}{2 \tau}\left[\operatorname{dist}(\bar{x}, C)-d\left(\bar{x}, a_{1}\right)\right] \\
\geq & \frac{1}{2 \tau}\left[\operatorname{dist}(\bar{x}, C)-\operatorname{dist}\left(\bar{x}, C_{1}\right)-\epsilon\right] \\
\geq & \frac{1}{2 \tau}\left[\frac{2 \tau}{2 \tau+1} \operatorname{dist}(\bar{x}, C)-\epsilon\right],
\end{aligned}
$$

where the last inequality holds thanks to (3.10). Letting $\epsilon \rightarrow 0$, we obtain that

$$
\operatorname{dist}(\bar{x}, C) \leq(2 \tau+1) \max \left\{\operatorname{dist}\left(\bar{x}, C_{1}\right), \operatorname{dist}\left(\bar{x}, C_{2}\right)\right\}
$$

which contradicts $(3.10)$.

Let $F: X \rightarrow 2^{Y}$ be a multifunction and let $G r(F)$ denote its gragh. Let $b \in Y$. Consider the problem of set inclusion:

$$
b \in F(x) .
$$

Thus $F^{-1}(b):=\{x \in X: b \in F(x)\}$ consists of all $x$ satisfying (3.11).

Definition 3.2. A function $\Upsilon:[0,+\infty) \rightarrow[0,+\infty)$ is called an admissible function if $\Upsilon$ is nondecreasing, $\Upsilon(0)=0$ and $\lim _{t \rightarrow 0} \Upsilon(t)=0$. The set of all admissible functions is denoted by $\Gamma$.

Part (b) of the following definitions was introduced and studied in [15] by $\mathrm{Li}$ and Singer for the special case when $X, Y$ are normed vector spaces; Lipschitz error bounds in this setup have been studied by them (see also [18, 24, 25]).

Definition 3.3. Let $\Upsilon \in \Gamma$ and let $F: X \rightarrow 2^{Y}, b \in Y$. We say that

(a) F has a $\Upsilon$-error bound for set inclusion $b \in F(x)$ if

$$
\operatorname{dist}\left(x, F^{-1}(b)\right) \leq \Upsilon[\operatorname{dist}(b, F(x))], \quad \forall x \in X .
$$


(b) F has a Lipschitz error bound for set inclusion $b \in F(x)$ if there exists $\tau>0$ such that

$$
\operatorname{dist}\left(x, F^{-1}(b)\right) \leq \tau \operatorname{dist}(b, F(x)), \quad \forall x \in X
$$

Remark 3.1. By considering $\Gamma(t)=\tau t$, (a) is seen to be a generalization of $(b)$.

Theorem 3.2. Suppose that $G r(F)$ is closed in $X \times Y$. Let $b \in Y$ be such that $F^{-1}(b) \neq \emptyset$. Then the following statements are equivalent:

(i) There exists $\Upsilon \in \Gamma$ such that $F: X \rightarrow 2^{Y}$ has a $\Upsilon$-error bound for set inclusion $b \in F(x)$.

(ii) $\lim _{k \rightarrow+\infty} \operatorname{dist}\left(x_{k}, F^{-1}(b)\right)=0$ whenever $\left\{x_{k}\right\} \subset X$ and $\lim _{k \rightarrow+\infty} \operatorname{dist}\left(b, F\left(x_{k}\right)\right)=0$.

(iii) The pair $\{G r(F), X \times\{b\}\}$ is regular.

Proof. Note that $F^{-1}(b) \times\{b\}=G r(F) \cap(X \times\{b\})$. Since $\operatorname{Gr}(F)$ is closed, so is $F^{-1}(b)$.

The proof of $(\mathrm{i}) \Rightarrow$ (ii) is straightforward from (3.12) as $\lim _{t \rightarrow 0} \Upsilon(t)=0$. The implication (iii) $\Rightarrow$ (ii) is also easily seen from definitions.

(ii) $\Rightarrow(\mathrm{i})$ : Define $\Upsilon(t):=\sup \left\{\operatorname{dist}\left(x, F^{-1}(b)\right): \operatorname{dist}(b, F(x)) \leq t\right\}$ for each $t \geq 0$. It is easy to verify that $\Upsilon(0)=0$ and $\Upsilon$ is a nondecreasing function such that (3.12) holds. To establish (i), it remains to show that $\lim _{t \rightarrow 0} \Upsilon(t)=0$. Let $\left\{t_{k}\right\} \subset(0,+\infty)$ be such that $\lim _{k \rightarrow+\infty} t_{k}=0$. By definition of $\Upsilon$, there exists $\left\{x_{k}\right\} \subset X$ satisfying $\operatorname{dist}\left(b, F\left(x_{k}\right)\right) \leq t_{k}$ such that $\Upsilon\left(t_{k}\right) \leq \operatorname{dist}\left(x_{k}, F^{-1}(b)\right)+\frac{1}{k}$ for each $k$. Then $\lim _{k \rightarrow+\infty} \operatorname{dist}\left(b, F\left(x_{k}\right)\right)=0$ and it follows from (ii) that $\lim _{k \rightarrow+\infty} \operatorname{dist}\left(x_{k}, F^{-1}(b)\right)=0$. Thus $\lim _{k \rightarrow+\infty} \Upsilon\left(t_{k}\right)=0$; and this implies that $\lim _{t \rightarrow 0} \Upsilon(t)=0$.

(ii) $\Rightarrow$ (iii): Suppose that (iii) is false. Then by Theorem 3.1 (applied to the pair $\{G r(F), X \times\{b\}\}$ of two closed sets in $X \times Y)$, there exist $\epsilon>0$ and $\left\{x_{k}\right\} \subset X$ such that

$$
\lim _{k \rightarrow+\infty} \operatorname{dist}\left(\left(x_{k}, b\right), G r(F)\right)=0,
$$

but $\operatorname{dist}\left(\left(x_{k}, b\right), \operatorname{Gr}(F) \cap(X \times\{b\})\right)>\epsilon$, that is

$$
\operatorname{dist}\left(x_{k}, F^{-1}(b)\right)>\epsilon, \quad \forall k \in \mathbb{N} .
$$

Let $\left(u_{k}, v_{k}\right) \in G r(F)$ be such that

$$
d\left(\left(u_{k}, v_{k}\right),\left(x_{k}, b\right)\right) \leq \operatorname{dist}\left(\left(x_{k}, b\right), G r(F)\right)+\frac{1}{k}, \quad \forall k \in \mathbb{N} .
$$

¿From (3.14), it follows that

$$
\lim _{k \rightarrow+\infty} d\left(x_{k}, u_{k}\right)=0
$$

and $\lim _{k \rightarrow+\infty} d\left(v_{k}, b\right)=0$, which implies that

$$
\lim _{k \rightarrow+\infty} \operatorname{dist}\left(b, F\left(u_{k}\right)\right)=0 .
$$

¿From (3.15) and (3.16) we have $\operatorname{dist}\left(u_{k}, F^{-1}(b)\right) \geq \frac{\epsilon}{2}$ for $k$ large enough. This and (3.17) together contradict (ii). 
Corollary 3.1. Let $f: X \rightarrow(-\infty,+\infty]$ be a proper lower semicontinuous function and let $S:=\{x \in X: f(x) \leq 0\}$. Suppose that $S$ is nonempty. Then the following statements are equivalent:

(i) There exists $\Upsilon \in \Gamma$ such that $f$ has a $\Upsilon$-error bound.

(ii) $\lim _{k \rightarrow+\infty} \operatorname{dist}\left(x_{k}, S\right)=0$ whenever $\left\{x_{k}\right\} \subset X$ and $\lim _{k \rightarrow+\infty} f\left(x_{k}\right)=0$.

(iii) The pair $\{\operatorname{epi}(f), X \times\{0\}\}$ is regular.

Proof. By assumption, epi $(f)$ is closed so the meaning of (iii) is defined by Definition 3.1. Define $F: X \rightarrow 2^{\mathbb{R}}$ by $F(x):=f(x)+[0,+\infty)$ for all $x \in X$. Then $G r(F)=\operatorname{epi}(f)$. It is easy to see that $S=F^{-1}(0)$ and that $\operatorname{dist}(0, F(x))=[f(x)]_{+}$for all $x \in X$. Thus the assertions follow immediately from Theorem 3.2.

\subsection{Normed Space Case}

For the remainder of this paper we need the vector structure. In particular, throughout this subsection, we assume that $X, Y$ are normed vector spaces (for the next section, $X$ and $Y$ will be required to be complete). For our convenience, we will use the norm for $X \times Y$ defined by $\|(x, y)\|=\sqrt{\|x\|^{2}+\|y\|^{2}}$ for all $(x, y) \in X \times Y$.

Lemma 3.1. Let $\tau>0$ and let $V_{\tau}$ be defined by $V_{\tau}=\{\underline{(x, y) \in X \times Y}: \tau\|x\| \leq\|y\|\}$. Let $S$ be a nonempty closed set in $Y, b \in Y$ and let $S^{\tau}=\overline{(S \times\{b\})+V_{\tau}}$. Then

$$
\operatorname{dist}\left((x, b), S^{\tau}\right)=\frac{\tau}{\sqrt{\tau^{2}+1}} \operatorname{dist}(x, S), \quad \forall x \in X
$$

Proof. Note first that $t V_{\tau} \subseteq V_{\tau}$ for any $t \geq 0$. Let $x \in X$ and $\epsilon>0$. Take $s \in S$ such that $\|x-s\| \leq \operatorname{dist}(x, S)+\epsilon$. Fix an element $e \in Y$ with $\|e\|=1$. Let $v:=\frac{1}{\tau^{2}+1}(x-s, \tau\|x-s\| e)$, and $u:=(s, b)+v$. Thus $v \in V_{\tau}, u \in S^{\tau}$, and

$$
u-(x, b)=(s-x, 0)+v=\left(\frac{-\tau^{2}}{\tau^{2}+1}(x-s), \frac{\tau\|x-s\|}{\tau^{2}+1} e\right) .
$$

Hence

$$
\|(x, b)-u\|=\frac{\tau}{\sqrt{\tau^{2}+1}}\|x-s\|
$$

and it follows that

$$
\operatorname{dist}\left((x, b), S^{\tau}\right) \leq \frac{\tau}{\sqrt{\tau^{2}+1}}\|x-s\| \leq \frac{\tau}{\sqrt{\tau^{2}+1}}(\operatorname{dist}(x, S)+\epsilon) .
$$

Since $\epsilon$ is arbitrary, this implies that

$$
\operatorname{dist}\left((x, b), S^{\tau}\right) \leq \frac{\tau}{\sqrt{\tau^{2}+1}} \operatorname{dist}(x, S), \quad \forall x \in X .
$$

To prove (3.18), suppose the strict inequality in (3.19) holds for some $x$. Then $x \notin S$ and there exist $s \in S,(z, y) \in V_{\tau}$ such that

$$
\|(x, b)-[(s, b)+(z, y)]\|<\frac{\tau}{\sqrt{\tau^{2}+1}} \operatorname{dist}(x, S) .
$$


It follows from the triangle inequality that

$$
(\|x-s\|-\|z\|)^{2}+\|y\|^{2}<\frac{\tau^{2}}{\tau^{2}+1} \operatorname{dist}(x, S)^{2} \leq \frac{\tau^{2}}{\tau^{2}+1}\|x-s\|^{2} .
$$

Letting $t=\frac{\|z\|}{\|x-s\|}$ and noting that $\|y\| \geq \tau\|z\|$, it follows that

$$
\|x-s\|^{2}(1-t)^{2}+\tau^{2} t^{2}\|x-s\|^{2}<\frac{\tau^{2}}{\tau^{2}+1}\|x-s\|^{2} .
$$

Since $x \neq s,(3.20)$ becomes

$$
\left(1+\tau^{2}\right) t^{2}-2 t+\frac{1}{\tau^{2}+1}<0
$$

which is clearly not possible.

The following result for multifunctions should be compared with Theorem 3.2: one for regularity while the other one is for linear regularity.

Theorem 3.3. Let $F: X \rightarrow 2^{Y}$ be a multifunction with closed graph $G r(F)$. Let $b \in Y$. Then the following two statements are equivalent:

(i) F has a Lipschitz error bound for set inclusion $b \in F(x)$.

(ii) $\{G r(F), X \times\{b\}\}$ is linearly regular.

Proof. Let $S:=F^{-1}(b)$. Then $S \times\{b\}=G r(F) \cap(X \times\{b\})$. If $\{G r(F), X \times\{b\}\}$ is linearly regular, then there exists $\kappa>0$ such that, for any $x \in X$,

$$
\begin{aligned}
\operatorname{dist}(x, S) & =\operatorname{dist}((x, b), S \times\{b\}) \\
& =\operatorname{dist}((x, b), G r(F) \cap(X \times\{b\})) \\
& \leq \operatorname{cdist}((x, b), G r(F)) .
\end{aligned}
$$

Since $\operatorname{dist}((x, b), G r(F)) \leq \operatorname{dist}((x, b),(x, F(x)))=\operatorname{dist}(b, F(x))$, we have (ii) $\Rightarrow(\mathrm{i})$.

Next we prove that (i) $\Rightarrow$ (ii). By assumption there exists $\tau>0$ such that

$$
\operatorname{dist}(x, S) \leq \tau \operatorname{dist}(b, F(x)), \forall x \in X .
$$

Let $V_{\tau}=\{(x, y) \in X \times Y:\|x\| \leq \tau\|y\|\}$ and $S^{\tau}=\overline{(S \times\{b\})+V_{\tau}}$ as in the preceding lemma. We will show that $\operatorname{Gr}(F) \subset S^{\tau}$. Since $(0, y-b) \in V_{\tau}$ for each $y \in Y$, we have $(x, y) \in S^{\tau}$ for each $x \in S$. For $x \notin S$ with $(x, y) \in G r(F)$, take $s_{n} \in S$ such that $\operatorname{dist}(x, S)+\frac{1}{n} \geq\left\|x-s_{n}\right\|$. Let $\sigma_{n}:=\frac{1}{n \operatorname{dist}(x, S)}$ and let $y_{n}:=y+\sigma_{n}(y-b)$. It is clear that $y_{n} \rightarrow y$. Also, by (3.21), $\operatorname{dist}(x, S) \leq \tau\|y-b\|$ and it follows that $\tau\left\|y_{n}-b\right\|=$ $\tau\left(1+\sigma_{n}\right)\|y-b\| \geq\left(1+\sigma_{n}\right) \operatorname{dist}(x, S) \geq \operatorname{dist}(x, S)+\frac{1}{n} \geq\left\|x-s_{n}\right\|$, which implies that $\left(x, y_{n}\right) \in S^{\tau}$, and hence that $(x, y) \in S^{\tau}$ because $S^{\tau}$ is closed. Therefore $\operatorname{Gr}(F) \subseteq S^{\tau}$. From Lemma 3.1, it follows that

$$
\begin{aligned}
\operatorname{dist}((x, b), S \times\{b\}) & =\operatorname{dist}(x, S) \\
& =\frac{\sqrt{\tau^{2}+1}}{\tau} \operatorname{dist}\left((x, b), S^{\tau}\right) \\
& \leq \frac{\sqrt{\tau^{2}+1}}{\tau} \operatorname{dist}((x, b), \operatorname{Gr}(F))
\end{aligned}
$$


By Theorem 3.1 (applied to the pair $\{G r(F), X \times\{b\}\}$ of two closed sets in $X \times Y$ ), this implies that (ii) holds.

Remark 3.2. Suppose that $G r(F) \subset \mathbb{R}^{n} \times \mathbb{R}^{m}$ is a polyhedron such that $G r(F) \cap\left(\mathbb{R}^{n} \times\right.$ $\{b\}) \neq \emptyset . \quad B y\left[4\right.$, Corollary 5.26], $\left\{G r(F), \mathbb{R}^{n} \times\{0\}\right\}$ is linearly regular. Hence the assertion [23, Example 9.47] that $F$ has a Lipschitz error bound follows immediately from Theorem 3.3.

Corollary 3.2. Let $f: X \rightarrow \mathbb{R}$ be a lower semicontinuous function. Then $f$ has a Lipschitz error bound if and only if $\{$ epi $(f), X \times\{0\}\}$ is linearly regular.

Proof. The proof is same as that for Corollary 3.1 except that one applies Theorem 3.3 in place of Theorem 3.2.

\section{Linear Regularity of Convex Sets in Banach Spaces}

The discussions of our works in what follows depends on some convexity assumptions. Thus we will assume throughout this section that our sets in the collection $\left\{C_{i}\right\}_{i=1}^{m}$ are convex in addition to the assumption that they are closed. Assume further that, for the following definition (and Theorem 4.1), each $C_{i}$ is a cone. The following definition is a reformulation of Jameson's property (G) (thanks to [6, Proposition 4 and Corollary 1]).

Definition 4.1. Let $X$ be a normed vector space and let $\left\{C_{i}\right\}_{i=1}^{m}$ be a collection of closed convex cones in $X$. The collection is said to have property $(G)$ if there exists $\alpha>0$ such that

$$
\inf \left\{\max _{1 \leq i \leq m}\left\|x_{i}\right\|: \text { each } x_{i} \in C_{i}, \sum_{i=1}^{m} x_{i}=x\right\} \leq \alpha\|x\|, \quad \forall x \in \sum_{i=1}^{m} C_{i} .
$$

The following result can be proved easily (recall our convention made in (2.8)).

Lemma 4.1. The collection $\left\{C_{i}\right\}_{i=1}^{m}$ of closed convex cones in $X$ has property $(G)$ if and only if there exist $\tau>0$ and $p \in[1,+\infty]$ such that

$$
\inf \left\{\left[\sum_{i=1}^{m}\left\|x_{i}\right\|^{p}\right]^{\frac{1}{p}}: \text { each } x_{i} \in C_{i}, \sum_{i=1}^{m} x_{i}=x\right\} \leq \tau\|x\| \quad \forall x \in \sum_{i=1}^{m} C_{i},
$$

(In the case of $(4.2)$ we will also say that $\left\{C_{i}\right\}_{i=1}^{m}$ has property $\left(G_{\tau, p}\right)$ ).

Remark 4.1. In the case when $X=Y^{*}$ for some Banach space $Y$ (that is $X$ is a Banach dual space) such that each $C_{i}$ is weak*-closed, the infimum in (4.2) is attained. In fact, for any $x \in \sum_{i=1}^{m} C_{i}$, let $\left\{x_{i}^{n}\right\} \subset Y^{*}$ be such that each $x_{i}^{n} \in C_{i}, \sum_{i=1}^{m} x_{i}^{n}=x$ and $\left[\sum_{i=1}^{m}\left\|x_{i}^{n}\right\|^{p}\right]^{\frac{1}{p}} \leq \tau\|x\|+\frac{1}{n}$. Then for any fixed $i \in[1, m], x_{i}^{n}$ is a bounded sequence in $Y^{*}$. By Alaoglu Theorem (cf. [12, p.70]) and considering subnets if necessary, there exist 
$x_{1}, \cdots, x_{m}$ such that $x_{i}^{n} \stackrel{w^{*}}{\rightarrow} x_{i}$ for each $i=1, \cdots, m$. It is clear that $x_{i} \in C_{i}$ and $\sum_{i=1}^{m} x_{i}=x$. By [16, Theorem 2.6.14], one has that $\left\|x_{i}\right\| \leq \liminf \left\|x_{i}^{n}\right\|$. This shows that the infimum in (4.2) is attained.

The proof given here for the following theorem is adopted from that given in $[6$, Proposition 6].

Theorem 4.1. Let $X$ be a Banach space, $\left\{C_{i}\right\}_{i=1}^{m}$ be a collection of closed convex cones in $X$ and let $C:=\cap_{i=1}^{m} C_{i}$. Let $\tau>0$ and $1 \leq p, q \leq+\infty$ with $\frac{1}{p}+\frac{1}{q}=1$. Then the following statements are equivalent:

(i) $\operatorname{dist}(z, C) \leq \tau\left[\sum_{i=1}^{m} \operatorname{dist}^{p}\left(z, C_{i}\right)\right]^{\frac{1}{p}}, \quad \forall z \in X$.

(ii) $C^{\circ}=\sum_{i=1}^{m} C_{i}^{\circ}$, and $\left\{C_{i}^{\circ}\right\}_{i=1}^{m}$ has property $\left(G_{\tau, q}\right)$, namely

$$
\min \left\{\left[\sum_{i=1}^{m}\left\|x_{i}^{*}\right\|^{q}\right]^{\frac{1}{q}}: \operatorname{each} x_{i}^{*} \in C_{i}^{\circ}, \sum_{i=1}^{m} x_{i}^{*}=x^{*}\right\} \leq \tau\left\|x^{*}\right\|, \quad \forall x^{*} \in \sum_{i=1}^{m} C_{i}^{\circ} .
$$

Proof. Clearly $\sum_{i=1}^{m} C_{i}^{\circ} \subseteq C^{\circ}$. We first consider the case when $p, q \in(1,+\infty)$. Set $g:=$ $\frac{1}{p} \operatorname{dist}^{p}(\cdot, C)=\frac{1}{p}\|\cdot\|^{p} \square \iota_{C}$. By Lemma 2.3, $g^{*}:=\frac{1}{q}\|\cdot\|^{q}+\iota_{C^{\circ}}$. Further set $f:=\sum_{i=1}^{m} f_{i}$ and $f_{i}:=\frac{\tau^{p}}{p} \operatorname{dist}^{p}\left(\cdot, C_{i}\right)$ for each $i$. By parts $(\mathrm{v})$ and $(i i i)$ of Lemma $2.3, f_{i}^{*}:=\frac{1}{q \tau^{q}}\|\cdot\|^{q}+\iota_{C_{i}^{\circ}}$. Indeed, for each $x^{*} \in X^{*}$,

$$
f_{i}^{*}\left(x^{*}\right)=\left(\frac{\tau^{p}}{p}\|\cdot\|^{p} \square \iota_{C}\right)^{*}\left(x^{*}\right)=\tau^{p}\left(\frac{1}{p}\|\cdot\|^{p}\right)^{*}\left(\frac{x^{*}}{\tau^{p}}\right)+\iota_{C_{i}^{\circ}}\left(x^{*}\right)=\frac{\tau^{p(1-q)}}{q}\left\|x^{*}\right\|^{q}+\iota_{C_{i}^{\circ}}\left(x^{*}\right) .
$$

Because each $f_{i}$ is everywhere continuous, it follows from Remark 2.1 that $f^{*}=f_{1}^{*} \square \ldots \square f_{m}^{*}$ and this infimal convolution is exact on $\sum_{i=1}^{m} C_{i}^{\circ}$. Using this, we obtain: (i) $\Leftrightarrow \mathrm{g} \leq \mathrm{f} \Leftrightarrow \mathrm{f}^{*} \leq$ $\mathrm{g}^{*} \Leftrightarrow \frac{1}{\mathrm{q} \tau^{\mathrm{q}}} \inf \left\{\sum_{\mathrm{i}=1}^{\mathrm{m}}\left\|\mathrm{x}_{\mathrm{i}}^{*}\right\|^{\mathrm{q}}: \forall \mathrm{x}_{\mathrm{i}}^{*} \in \mathrm{C}_{\mathrm{i}}^{\circ}, \sum_{\mathrm{i}=1}^{\mathrm{m}} \mathrm{x}_{\mathrm{i}}^{*}=\mathrm{x}^{*}\right\} \leq\left(\frac{1}{\mathrm{q}}\|\cdot\|^{\mathrm{q}}+\iota_{\mathrm{C}^{\circ}}\right)\left(\mathrm{x}^{*}\right), \forall \mathrm{x}^{*} \in \mathrm{X}^{*} \Leftrightarrow \mathrm{C}^{\circ}=$ $\sum_{\mathrm{i}=1}^{\mathrm{m}} \mathrm{C}_{\mathrm{i}}^{\circ}$ and $\min \left\{\sum_{i=1}^{m}\left\|x_{i}^{*}\right\|^{q}: \forall x_{i}^{*} \in C_{i}^{\circ}, \sum_{i=1}^{m} x_{i}^{*}=x^{*}\right\} \leq \tau^{q}\left\|x^{*}\right\|^{q} \forall x^{*} \in \sum_{i=1}^{m} C_{i}^{\circ} \Leftrightarrow$ (ii).

The case $p=1$ (and so $q=+\infty$ ): Assume that (i) holds. Let $\gamma>1$; take $p_{0}>1$ such that $m^{1-\frac{1}{p_{0}}}<\gamma$. By Lemma 2.4, it follows that $\sum_{i=1}^{m} \operatorname{dist}\left(z, C_{i}\right) \leq \gamma\left[\sum_{i=1}^{m} \operatorname{dist}^{p_{0}}\left(z, C_{i}\right)\right]^{\frac{1}{p_{0}}}$ for any $z \in X$. So $\operatorname{dist}(z, C) \leq \tau \gamma\left[\sum_{i=1}^{m} \operatorname{dist}^{p_{0}}\left(z, C_{i}\right)\right]^{\frac{1}{p_{0}}}$ for any $z \in X$. From the proof above, we have $C^{\circ}=\sum_{i=1}^{m} C_{i}^{\circ}$ and $\left\{C_{i}^{\circ}\right\}_{i=1}^{m}$ has property $\left(G_{\tau \gamma, q_{0}}\right)$, where $q_{0}$ satisfies $\frac{1}{p_{0}}+\frac{1}{q_{0}}=1$. That is, (4.3) holds for $\tau \gamma$ and $q_{0}$ in place of $\tau$ and $q$; hence it follows from Lemma 2.4 that (4.3) with $\tau \gamma$ in place of $\tau$ also holds for $q=+\infty$. Letting $\gamma \rightarrow 1$, (ii) is seen to hold (by Remark 4.1, the infimum is attained). Next, we prove that (ii) $\Rightarrow$ (i). For $\gamma>1$, 
by Lemma 2.4, there exists $q_{0}$ such that $\left[\sum_{i=1}^{m}\left\|x_{i}^{*}\right\|^{q_{0}}\right]^{\frac{1}{q_{0}}} \leq \gamma \max _{1 \leq i \leq m}\left\{\left\|x_{i}^{*}\right\|\right\}, \forall x_{i}^{*} \in X^{*}$. Then (ii) with $q=+\infty$ implies that $\left\{C_{i}^{\circ}\right\}_{i=1}^{m}$ has property $\left(G_{\tau \gamma, q_{0}}\right)$, and so, by what we have proved, $\operatorname{dist}(z, C) \leq \tau \gamma\left[\sum_{i=1}^{m} \operatorname{dist}^{p_{0}}\left(z, C_{i}\right)\right]^{\frac{1}{p_{0}}}$, where $p_{0}$ satisfies $\frac{1}{p_{0}}+\frac{1}{q_{0}}=1$. Letting $\gamma \rightarrow 1$, (i) is seen to hold.

The case $p=+\infty$ (and so $q=1$ ): By Lemma 2.4, (i) with $p=+\infty$ implies that $\operatorname{dist}(z, C) \leq \tau\left[\sum_{i=1}^{m} \operatorname{dist}^{p_{0}}\left(z, C_{i}\right)\right]^{\frac{1}{p_{0}}}$ for each $z \in X, \forall p_{0}>1$. Thus $C^{\circ}=\sum_{i=1}^{m} C_{i}^{\circ}$ and, by what we have proved, $\left\{C_{i}^{\circ}\right\}_{i=1}^{m}$ has property $\left(G_{\tau, q_{0}}\right)$ for any $1<q_{0}<+\infty$. Letting $q_{0} \rightarrow 1$, (ii) is seen to hold for $q=1$. Assume that (ii) holds for $q=1$. By Lemma 2.4, $\left\{C_{i}^{\circ}\right\}_{i=1}^{m}$ has property $\left(G_{\tau, q_{0}}\right)$ for any $q_{0}>1$. Then, by what we have proved, $\operatorname{dist}(z, C) \leq$ $\tau\left[\sum_{i=1}^{m} \operatorname{dist}^{p_{0}}\left(z, C_{i}\right)\right]^{\frac{1}{p_{0}}}, \forall z \in X$, and for any $p_{0} \in(1,+\infty)$. Letting $p_{0} \rightarrow 1$, we prove that (ii) $\Rightarrow$ (i).

Remark 4.2. Theorem 4.1 is valid (with the same proof) even if $X$ is a (not necessarily complete) normed vector space.

The objective of our next theorem (Theorem 4.2) is to extend the preceding theorem to the case when some $C_{i}$ is not necessarily a cone. As a preparatory step, we need the following lemma.

Lemma 4.2. Let $X$ be a Banach space and $C:=\cap_{i=1}^{m}\left\{C_{i}\right\}_{i=1}^{m} \neq \emptyset$, where each $C_{i}$ is a closed convex set in $X$. Let $\tau>0$, and $1 \leq p, q \leq+\infty$ with $\frac{1}{p}+\frac{1}{q}=1$. Then for any $z \in C$, the following statements are equivalent:

(i) $\operatorname{dist}\left(x, T_{C}(z)\right) \leq \tau\left[\sum_{i=1}^{m} \operatorname{dist}^{p}\left(x, T_{C_{i}}(z)\right)\right]^{\frac{1}{p}}, \quad \forall x \in X$.

(ii) The collection $\left\{C_{i}\right\}_{i=1}^{m}$ has the strong CHIP at $z$, and $\left\{N_{C_{i}}(z)\right\}$ has property $\left(G_{\tau, q}\right)$, namely

$$
\min \left\{\left[\sum_{i=1}^{m}\left\|x_{i}^{*}\right\|^{q}\right]^{\frac{1}{q}}: \operatorname{each} x_{i}^{*} \in N_{C_{i}}(z), \sum_{i=1}^{m} x_{i}^{*}=x^{*}\right\} \leq \tau\left\|x^{*}\right\|, \forall x^{*} \in \sum_{i=1}^{m} N_{C_{i}}(z) .
$$

Proof. Let $z \in C$. Note first that

$$
T_{C}(z)=\cap_{i=1}^{m} T_{C_{i}}(z)
$$

if (i) holds or if (ii) holds. Indeed, by taking polars, the above equality clearly holds if $\left\{C_{i}\right\}_{i=1}^{m}$ has the strong CHIP. If $v \in T_{C_{i}}(z)$ for all $i$, then $\operatorname{dist}\left(x, T_{C_{i}}(z)\right)=0$ and hence $\operatorname{dist}\left(x, T_{C}(z)\right)=0$ by (i). It follows that $T_{C}(z) \supseteq \cap_{i=1}^{m} T_{C_{i}}(z)$; thus (4.5) holds if (i) holds because $C \subseteq C_{i}$ for each $i$. Since $N_{C}(z)$ and $N_{C_{i}}(z)$ are respectively the polars of $T_{C}(z)$ and $T_{C_{i}}(z)$, the lemma is seen to hold by Theorem 4.1 (applied to $\left\{T_{C_{i}}(z)\right\}_{i=1}^{m}$ in place of $\left.\left\{C_{i}\right\}_{i=1}^{m}\right)$.

Remark 4.3. Notice that (i) is a linear regularity condition of $\left\{T_{C_{i}}(z)\right\}_{i=1}^{m}$. In what follows we will explore the possibility to place it by the linear regularity condition of the original collection $\left\{C_{i}\right\}_{i=1}^{m}$. 
The next theorem is one of our main results; it extends Theorem 4.1 to cover the case when some $C_{i}$ is not necessarily a cone.

Theorem 4.2. Let $X$ be a Banach space. Suppose that $C_{1}, \cdots, C_{m}$ are finitely many closed convex sets in $X$ with $C:=\cap_{i=1}^{m} C_{i} \neq \emptyset$. Let $\tau>0$ and $1 \leq p, q \leq+\infty$ with $\frac{1}{p}+\frac{1}{q}=1$. Then the following statements are equivalent:

(i) If $z \in C$, then $N_{C}(z)=\sum_{i=1}^{m} N_{C_{i}}(z)$ and

$$
\min \left\{\left[\sum_{i=1}^{m}\left\|z_{i}^{*}\right\|^{q}\right]^{\frac{1}{q}}, \text { each } z_{i}^{*} \in N_{C_{i}}(z), \sum_{i=1}^{m} z_{i}^{*}=z^{*}\right\} \leq \tau\left\|z^{*}\right\|, \quad \forall z^{*} \in N_{C}(z) .
$$

(ii) $\operatorname{dist}\left(x, z+T_{C}(z)\right) \leq \tau\left[\sum_{i=1}^{m} \operatorname{dist}^{p}\left(x, z+T_{C_{i}}(z)\right)\right]^{\frac{1}{p}}, \quad \forall x \in X, \forall z \in C$.

$\left(\mathrm{ii}^{*}\right) \operatorname{dist}\left(x, T_{C}(z)\right) \leq \tau\left[\sum_{i=1}^{m} \operatorname{dist}^{p}\left(x, T_{C_{i}}(z)\right)\right]^{\frac{1}{p}}, \quad \forall x \in X, \forall z \in C$.

(iii) $\operatorname{dist}(x, C) \leq \tau\left[\sum_{i=1}^{m} \operatorname{dist}^{p}\left(x, C_{i}\right)\right]^{\frac{1}{p}}, \quad \forall x \in X$.

Remark 4.4. Each of (i), (ii), (ii*) holds trivially for $z \in C \backslash b d(C)$.

Proof. By Lemma 4.2, (i) $\Leftrightarrow\left(\right.$ ii* $^{*}$. Certainly (ii) $\Leftrightarrow\left(\right.$ ii $\left.^{*}\right)$.

(ii) $\Rightarrow$ (iii). Let $x \in X$ and $\gamma \in(0,1)$. By Lemma 2.2, there exists $z \in C$ such that $\gamma \operatorname{dist}(x, C) \leq \operatorname{dist}\left(x, z+T_{C}(z)\right)$. Then $\gamma \operatorname{dist}(x, C) \leq \operatorname{dist}\left(x, z+T_{C}(z)\right) \leq$ $\tau\left[\sum_{i=1}^{m} \operatorname{dist}^{p}\left(x, z+T_{C_{i}}(z)\right)\right]^{\frac{1}{p}} \leq \tau\left[\sum_{i=1}^{m} \operatorname{dist}^{p}\left(x, C_{i}\right)\right]^{\frac{1}{p}}$. Hence (iii) is seen to hold.

(iii) $\Rightarrow$ (ii*). If not, then there exist $h \in X$ and $z_{0} \in C$ such that

$$
\operatorname{dist}\left(h, T_{C}\left(z_{0}\right)\right)>\tau\left[\sum_{i=1}^{m} \operatorname{dist}^{p}\left(h, T_{C_{i}}\left(z_{0}\right)\right)\right]^{\frac{1}{p}} .
$$

This implies in particular that $h \notin T_{C}\left(z_{0}\right)$. Let $x(t):=z_{0}+t h$ for $t>0$. Let $I$ consist of all $i$ satisfying the property that $h \in T_{C_{i}}\left(z_{0}\right)$. Then $I$ is not empty. For otherwise $h \notin T_{C_{i}}\left(z_{0}\right)$ and so $\operatorname{dist}\left(x(t), C_{i}\right)=o(t)$ for each $i$. It follows from (iii) that $\operatorname{dist}(x(t), C)=o(t)$, contradicting the fact that $h \notin T_{C}\left(z_{0}\right)$. Introducing the notation $\mathcal{F}(x(t)):=\frac{\left[\sum_{i \in I} \operatorname{dist}^{p}\left(x(t), z_{0}+T_{C_{i}}\left(z_{0}\right)\right]^{\frac{1}{p}}\right.}{\left[\sum_{i=1}^{m} \operatorname{dist}^{p}\left(x(t), C_{i}\right)\right]^{\frac{1}{p}}}$, note that

$$
\begin{aligned}
& \frac{\operatorname{dist}(x(t), C)}{\left[\sum_{i=1}^{m} \operatorname{dist}^{p}\left(x(t), C_{i}\right)\right]^{\frac{1}{p}}} \\
\geq & \frac{\operatorname{dist}(x(t), C)}{\operatorname{dist}\left(x(t), z_{0}+T_{C}\left(z_{0}\right)\right)} \frac{\operatorname{dist}\left(x(t), z_{0}+T_{C}\left(z_{0}\right)\right)}{\left[\sum_{i=1}^{m} \operatorname{dist}^{p}\left(x(t), z_{0}+T_{C_{i}}\left(z_{0}\right)\right)\right]^{\frac{1}{p}}} \mathcal{F}(x(t)), \\
= & \frac{\operatorname{dist}(x(t), C)}{\operatorname{dist}\left(x(t), z_{0}+T_{C}\left(z_{0}\right)\right)} \frac{t \operatorname{dist}\left(h, T_{C}\left(z_{0}\right)\right)}{t\left[\sum_{i=1}^{m} \operatorname{dist}^{p}\left(h, T_{C_{i}}\left(z_{0}\right)\right)\right]^{\frac{1}{p}}} \mathcal{F}(x(t)) .
\end{aligned}
$$


Since $\operatorname{dist}\left(x(t), C_{i}\right)=o(t)$ for each $i \notin I$ and $\lim _{t \rightarrow 0} \frac{\operatorname{dist}\left(x(t), z_{0}+T_{C_{i}}\left(z_{0}\right)\right)}{\operatorname{dist}\left(x(t), C_{i}\right)}=1$ for each $i \in I$ (see Lemma 2.1), one has $\lim _{t \rightarrow 0} \mathcal{F}(x(t))=1$. Noting also that $\lim _{t \rightarrow 0} \frac{\operatorname{dist}\left(x(t), z_{0}+T_{C}\left(z_{0}\right) \text { ) }\right.}{\operatorname{dist}(x(t), C)}=1$ (by Lemma 2.1), it follows from (4.6) and (4.7) that there exists $t^{\prime}>0$ such that

$$
\operatorname{dist}\left(x\left(t^{\prime}\right), C\right)>\tau\left[\sum_{i=1}^{m} \operatorname{dist}^{p}\left(x\left(t^{\prime}\right), C_{i}\right)\right]^{\frac{1}{p}} .
$$

This contradicts (iii).

Remark 4.5. In the special case when $X$ is an Euclidean space, some partial results of Theorem 4.2 are already known in the literature: (i) $\Rightarrow$ (iii) was proved in [6, Corollary 4] when $X=\mathbb{R}^{m}$ with $p=2$. While a part of (iii) $\Rightarrow(\mathrm{i})$ (the assertion that the strong CHIP follows from the linear regularity) was observed in [21, Proposition 6] (based on [14]) and in [6, Theorem 3] when $X=\mathbb{R}^{m}$ with $p=2$. We note also that [6, Theorem 1] follows easily from Theorem 4.2 and Hoffman's result.

In the remainder of this paper we will focus on the study of linear regularity for a pair of two closed convex sets and one of them is a closed subspace. We begin with the special case when the other closed convex set in the pair is in fact a cone. Thus, let $C_{1}=W$ be a closed vector space of $X$ and let $C_{2}$ be a closed convex cone in $X$, where $X$ is a Banach space. Define $P: X^{*} \rightarrow W^{*}$ by

$$
P\left(x^{*}\right)(w)=x^{*}(w), \quad \forall w \in W .
$$

That is, $P\left(x^{*}\right)$ is the restriction of $x^{*}$ to $W$.

Given $C_{2}$, one can define a preorder in $X$ by

$$
x \leq y \Leftrightarrow y-x \in C_{2} .
$$

Then $-C_{2}^{\circ}$ consists of all positive continuous linear functionals on $X$. Let $C:=C_{2} \cap W$, and let $C^{\circ} \cap W^{*}$ denote the polar of $C$ taken in $W^{*}$ :

$$
C^{\circ} \cap W^{*}=\left\{w^{*} \in W^{*}: w^{*}(c) \leq 0, \forall c \in C\right\} .
$$

Thus $-\left(C^{\circ} \cap W^{*}\right)$ consists of all positive continuous linear functionals on $W$. In view of Theorem 3.1 (b), the following result establishes a characterization for $\left\{W, C_{2}\right\}$ to be linearly regular. Also, for $c \in C$, in addition to the notion of the normal cone $N_{C}(c)=$ $\left(T_{C}(c)\right)^{\circ}$, let $N_{C}(c) \cap W^{*}$ denote the normal cone of $C$ at $c$ relative to $W$. That is,

$$
N_{C}(c) \cap W^{*}=\left\{w^{*} \in W^{*}: w^{*}(h) \leq 0, \forall h \in T_{C}(c)\right\} .
$$

Theorem 4.3. Let $C_{2}$ be a closed convex cone in a Banach space $X$ with the preorder defined by (4.9). Let $W$ be a closed subspace of $X$ and $C:=C_{2} \cap W$. Let $\tau>0$. Then the following statements are equivalent:

(i) For any $w^{*} \in\left(C^{\circ} \cap W^{*}\right), \min \left\{\left\|z^{*}\right\|: P\left(z^{*}\right)=w^{*}, z^{*} \in C_{2}^{\circ}\right\} \leq \tau\left\|w^{*}\right\|$.

(ii) For any $x^{*} \in C^{\circ}, \min \left\{\left\|z^{*}\right\|: P\left(z^{*}\right)=P\left(x^{*}\right), z^{*} \in C_{2}^{\circ}\right\} \leq \tau\left\|x^{*}\right\|$. 
(iii) $\operatorname{dist}\left(x, C_{2} \cap W\right) \leq \tau \operatorname{dist}\left(x, C_{2}\right), \forall x \in W$.

Proof. First, we show that (i) $\Leftrightarrow$ (ii). For any $x^{*} \in C^{\circ}$, it is obvious that $w^{*}=P\left(x^{*}\right) \in$ $\left(C^{\circ} \cap W^{*}\right),\left\|w^{*}\right\| \leq\left\|x^{*}\right\|$, and so (i) $\Rightarrow$ (ii). For $w^{*} \in\left(C^{\circ} \cap W^{*}\right)$, by the Hahn-Banach Theorem there exists $x^{*} \in X^{*}$ such that $P\left(x^{*}\right)=w^{*},\left\|x^{*}\right\|=\left\|w^{*}\right\|$. In particular, since $C \subset W$, one has that $x^{*}(c)=w^{*}(c) \leq 0$ for each $c \in C$. Thus, $x^{*} \in C^{\circ}$ and so (ii) $\Rightarrow$ (i).

Let $f$ and $g$ be defined by $f(x)=\frac{1}{2 \tau^{2}} \operatorname{dist}^{2}(x, C)$ and $g(x)=\frac{1}{2} \operatorname{dist}^{2}\left(x, C_{2}\right)+\iota_{W}$ for each $x \in X$. Thus (iii) reads that $f \leq g$. Moreover, by Lemma $2.3, f^{*}=\frac{\tau^{2}}{2}\|\cdot\|^{2}+\iota_{C^{\circ}}$, $g^{*}=\left(\frac{1}{2}\|\cdot\|^{2}+\iota_{C_{2}^{\circ}}\right) \square \iota_{W^{\circ}}$ and the infimal convolution $g^{*}$ is exact (for the latter we need (vi) of Lemma 2.3, which is applicable as $\operatorname{dist}^{2}\left(\cdot, C_{2}\right)$ is continuous). In particular, for any $x^{*} \in C_{2}^{\circ}+W^{\circ}$,

$$
g^{*}\left(x^{*}\right)=\min \left\{\frac{1}{2}\left\|z^{*}\right\|^{2}: z^{*} \in C_{2}^{\circ}, P\left(z^{*}\right)=P\left(x^{*}\right)\right\} .
$$

Thus, (iii) $\Leftrightarrow \mathrm{f} \leq \mathrm{g} \Leftrightarrow \mathrm{g}^{*} \leq \mathrm{f}^{*} \Leftrightarrow \mathrm{C}^{\circ}=\mathrm{C}_{2}^{\circ}+\mathrm{W}^{\circ}$ and $\min \left\{\left\|\mathrm{y}^{*}\right\|: \mathrm{y}^{*} \in \mathrm{C}_{2}^{\circ}, \mathrm{P}\left(\mathrm{y}^{*}\right)=\right.$ $\left.\mathrm{P}\left(\mathrm{x}^{*}\right)\right\} \leq \tau\left\|\mathrm{x}^{*}\right\|, \forall \mathrm{x}^{*} \in \mathrm{C}_{2}^{\circ}+\mathrm{W}^{\circ} \Leftrightarrow \min \left\{\left\|\mathrm{z}^{*}\right\|: \mathrm{z}^{*} \in \mathrm{C}_{2}^{\circ}, \mathrm{P}\left(\mathrm{z}^{*}\right)=\mathrm{P}\left(\mathrm{x}^{*}\right)\right\} \leq \tau\left\|\mathrm{x}^{*}\right\|, \forall \mathrm{x}^{*} \in$ $\mathrm{C}^{\circ} \Leftrightarrow$ (ii).

Theorem 4.4. Let $C_{2}$ be a closed convex set (not necessarily a cone) in $X$ such that $C:=C_{2} \cap W \neq \emptyset$, where $X$ and $W$ are as in the preceding theorem. For any $c \in C$, $N_{C}(c) \cap W^{*}$ is defined as in (4.10). Let $\tau>0$. Then the following statements are equivalent:

(i) If $z \in C$ and $w^{*} \in N_{C}(z) \cap W^{*}$, then

$$
\min \left\{\left\|z^{*}\right\|, P\left(z^{*}\right)=w^{*} \text { and } z^{*} \in N_{C_{2}}(z)\right\} \leq \tau\left\|w^{*}\right\|
$$

(ii) $\operatorname{dist}\left(w, z+T_{C}(z)\right) \leq \tau \operatorname{dist}\left(w, z+T_{C_{2}}(z)\right), \quad \forall w \in W, z \in C$.

(ii*) $\operatorname{dist}\left(w, T_{C}(z)\right) \leq \tau \operatorname{dist}\left(w, T_{C_{2}}(z)\right), \quad \forall w \in W, z \in C$.

(iii) $\operatorname{dist}(x, C) \leq \tau \operatorname{dist}\left(x, C_{2}\right), \forall x \in W$.

Proof. Certainly (ii) $\Leftrightarrow\left(\right.$ ii $^{*}$ ). We can prove (ii) $\Leftrightarrow$ (iii) similarly as in Theorem 4.2. It remains to show that $(\mathrm{i}) \Leftrightarrow\left(\mathrm{ii}^{*}\right)$. To this end, note first that provided (i) or (ii*) holds, $T_{C}(z)=T_{C_{2}}(z) \cap W$ for any $z \in C$. To verify this, we need only to show that

$$
T_{C}(z) \supseteq T_{C_{2}}(z) \cap W
$$

This inclusion certainly follows from (ii*). Next, we assume that (i) holds. Let $v \in$ $T_{C_{2}}(z) \cap W$, and let $w^{*} \in\left(N_{C}(z) \cap W^{*}\right)$. By (i), take $z^{*} \in N_{C_{2}}(z)$ such that $P\left(z^{*}\right)=w^{*}$. Then $w^{*}(v)=z^{*}(v) \leq 0$. Since $N_{C}(z) \cap W^{*}$ is the polar of $T_{C}(z)$ in the dual pair of $W$ and $W^{*}$, by the Bipolar Theorem, it follows that $v \in T_{C}(z)$. Therefore (4.12) holds, and so does the equality provided that (i) or (ii*) holds.

Since $N_{C_{2}}(z)=\left(T_{C_{2}}(z)\right)^{\circ}$ for each $z \in C$, (i) $\Leftrightarrow\left(\right.$ ii $\left.^{*}\right)$ now follows from Theorem 4.3 (applied to the pair $\left\{T_{C_{2}}(z), W\right\}$ in place of $\left\{C_{2}, W\right\}$ ). 


\section{References}

[1] H. Attouch and H. Brezis, Duality for the sum of convex functions in general Banach spaces, Aspects of Mathematics and Its applications, J. A. Barroso, eds., NorthHolland, Amsterdam, 1986, 125-133.

[2] J.-P., Aubin and I. Ekeland, Applied Nonlinear Analysis, Pure and Applied Mathematics, New York, 1984.

[3] H. H. Bauschke, Projection Algorithms and Monotone Operators. Ph.D thesis, Simon Fraser University, Department of Mathematics, Burnaby, British Columbia V5A 1S6, Canada, August 1996. Available at http://www.cecm.sfu.ca/preprints/1996pp.html

[4] H. H. Bauschke and J. M. Borwein, On projection algorithms for solving convex feasibility problems. SIAM Rev., 38(1996), 367-426.

[5] H. H. Bauschke and J. M. Borwein, On the convergence of von Neumann's alternating projection algorithm for two sets, Set-Valued Anal. 1(1993), 185-212.

[6] H. H. Bauschke, J. M. Borwein and W. Li, Strong conical hull intersection property, bounded linear regularity, Jameson's property $(G)$, and error bounds in convex optimization. Math. Program., 86(1999), 135-160.

[7] F. H. Clarke, Optimization and Nonsmooth Analysis, Wiley, New York, 1983.

[8] F. Deutsch, W. Li, and J. D. Ward, A dual approach to constrained interpolation from a convex subset of Hilbert space, J. Approx. Theory, 90(1997), 385-414.

[9] F. Deutsch, W. Li, and J. D. Ward, Best approximation from the intersection of a closed convex set and a polyhedron in Hilbert space, weak Slater conditions, and the strong conical hull intersection property, SIAM J. Optim. 10(1999), 252-268.

[10] F. Hirsch and L. Gilles, Elements of Functional Analysis, Springer-Verlag, New York, 1999.

[11] A. J. Hoffman, On approximate solutions of systems of linear inequalities, Journal of Research of the National Bureau of Standards, 49(1952), 263-265.

[12] R. B. Holmes, Geometric Functional Analysis and its Applications, Springer-Verlag, New York-Heidelberg, 1975.

[13] G. J. O. Jameson, The duality of pairs of wedges, Proc. Lond. Math. Soc., 24(1972), 531-547.

[14] A. Lewis and J.-S. Pang, Error bounds for convex inequality systems, in Generalized Convexity, Generalized Monotonicity: Recent Results, Proceedings of the Fifth Symposium on Generalized Convexity, Luminy, June 1996, J.-P. Crouzeix, J.-E. MartinezLegaz, and M.Volle, eds., Kluwer Academic Publishers, Dordrecht, 1997, 75-100.

[15] W. Li and I. Singer, Global error bounds for convex multifunctions and applications, Math. Oper. Res., 23(1998), 443-462. 
[16] R. E. Megginson, An Introduction to Banach Space Theory, Springer-Verlag, New York, 1998.

[17] K. F. Ng and X. Y. Zheng, Error bound for lower semicontinuous functions in normed spaces, SIAM J. Optim., 12(2001), 1-17.

[18] K. F. Ng and X. Y. Zheng, Characterizations of error bounds for convex multifunctions on Banach spaces, Preprint.

[19] K. F. Ng and W. H. Yang, Error bounds for abstract linear inequality systems, SIAM J. Optim., 13(2002), 24-43.

[20] K. F. Ng and W. H. Yang, Error bounds relating to quadratic cones, preprint.

[21] J.-S. Pang, Error bounds in mathematical programming, Math. Program., 79(1997), 299-332.

[22] R. T. Rockafellar, Convex Analysis, Princeton Univeersity Press, Princeton, NJ, 1970.

[23] R. T. Rockafellar and R. J.-B. Wets, Variational Analysis, Springer-Verlag, Berlin, 1998.

[24] C. Zalinescu, Nonlinear extension of Hoffman's error bound, to appear in Math. Oper. Res.

[25] X. Y. Zheng, Error bounds for set inclusions, to appear in Science in China. 